\title{
Mass Distribution in Space-Time and Conception about Space-Time-Mass Continuum
}

\author{
Rahul Singh \\ Department of Science and Technology National Library, Kolkata, India \\ Email: singhrahul.iit@gmail.com
}

How to cite this paper: Singh, R. (2018) Mass Distribution in Space-Time and Conception about Space-Time-Mass Continuum. International Journal of Astronomy and Astrophysics, 8, 94-103.

https://doi.org/10.4236/ijaa.2018.81007

Received: December 31, 2017

Accepted: March 19, 2018

Published: March 22, 2018

Copyright $\odot 2018$ by author and Scientific Research Publishing Inc. This work is licensed under the Creative Commons Attribution International License (CC BY 4.0).

http://creativecommons.org/licenses/by/4.0/

\begin{abstract}
There is a relation between space and time, which is called space-time continuum. With the help of some mathematical equations, we can establish an alternative relation between space-time and mass, treating mass as a fifth dimension. There is the concept about mass being a property of any inertial or gravitational object in space. Mass can create distortion in space. As a result of that, space-time curvature will be bent towards the massive object.
\end{abstract}

\section{Keywords}

Space-Time Curvature, Horizon Distance, Horizon Volume, Mass Distortion

\section{Introduction}

Mass is the part of dynamic process of gravitational interaction. It is an intrinsic property of celestial object [1]. Mass plays an important role in energy distribution in space-time. Mass prevails to establish the equation of energy-mass interchange [2]. Because of that, the space is accessible on the basis of mass-energy distribution. Energy is a form of mass with some limiting condition. The body with huge mass is also a great source of energy. Distribution of mass in space is similar to the distribution of energy with some extant. About Space-time-mass continuum with the mathematical changes in equations in Section 2 mass distribution in space-time in Section 3, what would be change by mass in space in Section 4, the relation between energy and temperature would be shown in Section 5 .

\section{Relation between Space and Mass}

As space and time are dimension of continuum with the magnitude of 4 . Now it 
is known as Minkowski space in which space and time is abbreviated in a single term with the three special and one time dimension. Here is the equation for space-time. Which was developed by Albert Einstein which compiled three space dimension with single time by a mathematical formulation of space-time continuum:

$$
(\Delta s)^{2}=(\Delta c t)^{2}-(\Delta x)^{2}-(\Delta y)^{2}-(\Delta z)^{2}
$$

where $(\Delta s)^{2}$ is space-time interval. $(\Delta x)^{2},(\Delta y)^{2},(\Delta z)^{2}$ are special components and $(\Delta c t)^{2}$ is time [3].

To make science more interesting mass was introduced when mass will be a line with the space-time. Then it will be a five dimensional matrix universe and the line element in five dimensional Euclidean flat space-time in spherical coordinate system with mass as the fifth dimension can be written down as-

$$
\mathrm{d} s^{2}=-\left(\mathrm{d} r^{2}+r^{2} \mathrm{~d} \theta^{2}+r^{2} \sin ^{2} \theta \mathrm{d} Q^{2}\right)+(c \mathrm{~d} t)^{2}+\left[(G / C)^{2} \mathrm{~d} m\right]^{2}
$$

the most general line element being:

$$
\mathrm{d} s^{2}=-\left(\mathrm{e}^{\lambda} \mathrm{d} r^{2}+r^{2} \mathrm{~d} \theta^{2}+r^{2} \sin ^{2} \theta \mathrm{d} \varphi^{2}\right)+\mathrm{e}^{v}(c \mathrm{~d} t)^{2}+\mathrm{e}^{\mu}\left[\left(\frac{G}{C^{2}}\right) \mathrm{d} m\right]^{2}
$$

where $\left(G / C^{2}\right)$ is the conversion factor which is a universal constant converting mass into length. Note that its value is of the order $10^{-26}$ (By this order the fifth dimension being suppressed). It has an effective consequence in the region containing higher mass [4].

The fifth dimension in five dimensional universe might be revealed to an appreciable level to begin with, e take $\lambda=\lambda(r), v=v(r)$ and $\mu=\mu(r)$ as function of $r$ alone, and at large distance from the attracting point mass sitting at the origin of the five-dimension universe. $\lambda, v, \mu \rightarrow 0$, as $r \rightarrow \infty$ to coincide with flat space-time-mass metric Equation (1).

The gravitational potentials $g_{i, j}(i, j=1,2,3,4,5)$ are covariant metric tensor given by

$$
\begin{aligned}
& {\left[g_{i, j}\right]=\left[\begin{array}{cccccc}
-\mathrm{e}^{\lambda} & 0 & 0 & & \\
0 & -r^{2} & 0 & & & \\
0 & 0 & -r^{2} \sin ^{2} & \theta & 0 & 0 \\
0 & 0 & 0 & 0 & 0 \\
0 & 0 & 0 & 0 & 0 \\
& & & & e^{v} & 0 \\
& & & 0 & e^{\mu}
\end{array}\right]} \\
& a=\left|g_{i, j}\right|, i, j=1,2,3,4,5
\end{aligned}
$$

The covariant metric tensor $g^{i j}$ are given by

$$
\left[g^{i j}\right]=\left[\begin{array}{ccccc}
-\mathrm{e}^{-\lambda} & 0 & 0 & 0 & 0 \\
0 & -r^{-2} & 0 & 0 & 0 \\
0 & 0 & -r^{-2} \sin ^{-2} \theta & 0 & 0 \\
0 & 0 & 0 & \mathrm{e}^{-v} & 0 \\
0 & 0 & 0 & 0 & \mathrm{e}^{-\mu}
\end{array}\right]
$$


The Christoffel symbols of the second kind are given by $\Gamma_{j k}^{i}=g^{i l}[j k, l]$, where $[j k, l]$ are the Christoffel symbols of the first kind and are being given by

$$
[j k, l]=\left(\frac{1}{2}\right)\left(g_{i j, k}+g_{k l, j}+g_{j k, l}\right)
$$

And $g^{i l}$ is contravariant metric tensor. We have $i, j, k=1,2,3,4,5$

There are 16 non-vanishing Christoffel symbols of second kind, which are givn below:

$$
\begin{aligned}
& \Gamma_{11}^{1}=\frac{\lambda^{\prime}}{2}, \Gamma_{22}^{1}=-r \mathrm{e}^{-\lambda}, \Gamma_{33}^{1}=-\mathrm{e}^{-\lambda} r \sin ^{2} \theta, \\
& \Gamma_{44}^{1}=\left(\frac{1}{2}\right) v^{\prime} \mathrm{e}^{\nu-\lambda}, \Gamma_{55}^{1}=\left(\frac{1}{2}\right) \mu^{\prime} \mathrm{e}^{\mu-\nu}, \\
& \Gamma_{12}^{2}=\Gamma_{21}^{2}=\frac{1}{r}, \Gamma_{33}^{2}=-\sin \theta \cos \theta, \\
& \Gamma_{13}^{3}=\Gamma_{23}^{3}=\frac{1}{r}, \Gamma_{32}^{3}=\cot \theta, \\
& \Gamma_{14}^{4}=\Gamma_{41}^{4}=\frac{v^{\prime}}{2}, \Gamma_{15}^{5}=\Gamma_{51}^{5}=\frac{\mu^{\prime}}{2}
\end{aligned}
$$

Einstein's field equations are:

$$
G_{i j}=0, i, j=1,2,3,4,5
$$

where $G_{i j}$ are given by:

$$
\begin{aligned}
G_{i j} \equiv & \left(\frac{\partial^{2}}{\partial^{i} \partial^{j}}\right)(\log \sqrt{-g})-\left(\frac{\partial}{\partial^{k}}\right) \Gamma_{i j}^{k}-\Gamma_{i j}^{l}\left(\frac{\partial}{\partial^{l}}\right)(\log \sqrt{-g}) \\
& +\Gamma_{i j}^{k} \Gamma_{i j}^{k}+\cdots, i, j=1,2,3,4,5
\end{aligned}
$$

From Equations (6)-(8), we get:

$$
\begin{gathered}
\left(\frac{v^{\prime \prime}}{2}\right)+\left(\frac{\mu^{\prime \prime}}{2}\right)-\left(\frac{\lambda^{\prime}}{r}\right)\left(v^{\prime}+\mu^{\prime}\right)-\left(\frac{\lambda^{\prime}}{r}\right)+\left(\frac{\left(v^{\prime}\right)^{2}+\left(\mu^{\prime}\right)^{2}}{4}\right)=0 \\
\mathrm{e}^{-\lambda}\left[1-\left(\frac{r \lambda^{\prime}}{2}\right)+\left(\frac{r}{2}\right)\left(v^{\prime}+\mu^{\prime}\right)\right]-1=0 \\
\sin ^{2} \theta\left[\mathrm{e}^{-\lambda}\left\{1-\left(\frac{r \lambda^{\prime}}{2}\right)+\left(\frac{r}{2}\right)\left(v^{\prime}+\mu^{\prime}\right)\right\}-1\right]=0 \\
\mathrm{e}^{(\nu-\lambda)}\left[\left(\frac{\lambda^{\prime} v^{\prime}}{4}\right)+\left(\frac{v^{\prime \prime}}{2}\right)-\left(\frac{v^{\prime}}{r}\right)-\left(\frac{v^{\prime 2}}{4}\right)-\left(\frac{v^{\prime} \mu^{\prime}}{4}\right)\right]=0 \\
\mathrm{e}^{(\mu-\lambda)}\left[\left(\frac{\mu^{\prime} \lambda^{\prime}}{4}\right)-\left(\frac{\mu^{\prime \prime}}{2}\right)-\left(\frac{\mu^{\prime}}{r}\right)-\left(\frac{\mu^{\prime 2}}{4}\right)-\left(\frac{v^{\prime} \mu^{\prime}}{4}\right)\right]=0 \\
G_{i j} \equiv 0, i \neq j, i, j=1,2,3,4,5
\end{gathered}
$$

These are equations in $r$ alone. They are difficult to solve in their very general form. We, therefore begin with the following simple cases:

Case (1): $\mu=\mu(r)=$ constant (say) $k$. As $\mu=\mu(r) \rightarrow 0$, as $r \rightarrow \infty$

This constant must be zero. In this simple case, 
The Equations 9(a)-(f) reduce to following:

$$
\begin{gathered}
\left(\frac{v^{\prime \prime}}{2}\right)-\left(\frac{\lambda^{\prime} v^{\prime}}{4}\right)-\left(\frac{\lambda^{\prime}}{r}\right)+\left(\frac{v^{\prime 2}}{4}\right)=0 \\
\mathrm{e}^{-\lambda}\left[1-\left(\frac{r \lambda^{\prime}}{2}\right)+\left(\frac{r v^{\prime}}{2}\right)\right]-1=0 \\
\sin ^{2} \theta\left[\mathrm{e}^{-\lambda}\left\{1-\left(\frac{r \lambda^{\prime}}{2}\right)+\left(\frac{r v^{\prime}}{2}\right)\right\}-1\right]=0 \\
\mathrm{e}^{(\nu-\mu)}\left[\left(\frac{\lambda^{\prime} v^{\prime}}{4}\right)-\left(\frac{v^{\prime \prime}}{2}\right)-\left(\frac{v^{\prime}}{r}\right)-\left(\frac{v^{\prime 2}}{4}\right)\right]=0 \\
G_{55} \equiv 0 \\
G_{i j} \equiv 0, i \neq j, i, j=1,2,3,4,5
\end{gathered}
$$

These are the Einstein's field equations in five dimensional universe with mass as its fifth dimension.

The solution of these equations is:

$$
\begin{aligned}
\mathrm{d} s^{2}= & -\left[\left\{1-\left(\frac{2 G M}{r c^{2}}\right)\right\}^{-1} \mathrm{~d} r^{2}+r^{2} \mathrm{~d} \theta^{2}+r^{2} \sin ^{2} \theta \mathrm{d} \varphi^{2}\right] \\
& +\left\{1-\left(\frac{2 G M}{r c^{2}}\right)\right\}(c \mathrm{~d} t)^{2}+\left[\left(\frac{G}{c^{2}}\right) \mathrm{d} m\right]^{2}
\end{aligned}
$$

If the mass is small, the mass dimension to be suppressed the conversion factor $\left(\frac{G}{c^{2}}\right)$, which is of the order of $10^{-26}$. Therefore in this case, we get Schwatzchild-like line element (solution) [5].

In five dimensional universe with the mass as its fifth dimension without showing much effect of mass dimension, except that it appears as one of the dimension in line element.

Case (2): $v=v(r)=$ constant (say) $k^{\prime}$. As $v=v(r) \rightarrow \infty$, this constant must be zero.

$$
\begin{gathered}
\left(\frac{\mu^{\prime \prime}}{2}\right)-\left(\frac{\lambda^{\prime} \mu^{\prime}}{4}\right)-\left(\frac{\lambda^{\prime}}{4}\right)+\left(\frac{\mu^{\prime 2}}{4}\right)=0 \\
\mathrm{e}^{-\lambda}\left[1-\left(\frac{r \lambda^{\prime}}{2}\right)+\left(\frac{r \mu^{\prime}}{2}\right)\right]-1=0 \\
\sin ^{2} \theta\left[\mathrm{e}^{-\lambda}\left\{1-\left(\frac{r \lambda^{\prime}}{2}\right)+\left(\frac{r \mu^{\prime}}{2}\right)\right\}-1\right]=0 \\
\mathrm{e}^{(\nu-\lambda)}\left[\left(\frac{\lambda^{\prime} \mu^{\prime}}{4}\right)-\left(\frac{\mu^{\prime \prime}}{2}\right)-\left(\frac{\mu^{\prime}}{r}\right)-\left(\frac{\mu^{\prime 2}}{4}\right)\right]=0 \\
G_{44} \equiv 0 \\
G_{i j} \equiv 0, i \neq j, i, j=1,2,3,4,5
\end{gathered}
$$


The solution of these equations is:

$$
\begin{aligned}
\mathrm{d} s^{2}= & -\left[\left\{1-\left(\frac{2 G M}{r c^{2}}\right)\right\}^{-1} \mathrm{~d} r^{2}+r^{2} \mathrm{~d} \theta^{2}+r^{2} \sin ^{2} \theta \mathrm{d} \varphi^{2}\right]+(c \mathrm{~d} t)^{2} \\
& +\left\{1-\left(\frac{2 G M}{r c^{2}}\right)\right\}\left[\left(\frac{G}{c^{2}}\right) \mathrm{d} m\right]^{2}
\end{aligned}
$$

Take mass, which also includes energy due to Einstein's energy-mass equivalence relation: $E=m c^{2}$, as the fifth dimension of universe.

This equation explains the co-relation of space-time with mass. Mass is the considerable aspect of universe. Dark matter and Dark energy have the equal role in space expansion [6] [7]. There is equivalent distribution of mass of radiation. Mass of matter and equivalent mass of dark energy within the horizon-volume of universe in the closed model of the universe are also participating in space expansion [8]. This model of space expansion elaborates the mass distribution with space expansion.

\section{The Distribution of Mass in Space with the Help of Cosmological Horizon}

The proper distance of an extragalactic object at present time is given by:

$$
d_{p}\left(t_{0}\right)=R\left(t_{0}\right) f\left(r_{0}\right)
$$

where $r_{0}$ is the comoving coordinate of the object now, and $f\left(r_{0}\right)$ is expressed as:

$$
f\left(r_{0}\right)=c \int_{t_{e}}^{t_{0}} \frac{\mathrm{d} t}{R(t)}=\int_{0}^{r_{0}} \frac{\mathrm{d} r}{\sqrt{1-k r^{2}}}= \begin{cases}r_{0} & (k=0) \\ \sin ^{-1} r_{0} & (k+1) \\ \sinh ^{-1} r_{0} & (k=-1)\end{cases}
$$

where $R(t)$ is the scale factor of the universe expansion, $c$ the speed of light, $t_{e}$ the emission time of object photon and $k$ the curvature of space in universe.

Let $a(t)=\frac{R(t)}{R\left(t_{0}\right)}$ then Equation (15) becomes

$$
f\left(r_{0}\right)=\frac{C}{R\left(t_{0}\right)} \int_{t_{e}}^{t_{0}} \frac{\mathrm{d} t}{a(t)}
$$

Substituting by Equation (16) in Equation (14) we get

$$
d_{p}\left(t_{0}\right)=c \int_{t_{e}}^{t_{0}} \frac{\mathrm{d} t}{a(t)}
$$

From [8], we have seen that the expansion speed of the universe is:

$$
a(t)=H_{0}\left[1-\Omega_{\Lambda, 0}\left(1-a^{2}\right)+\Omega_{m, 0}\left(\frac{1}{a}-1\right)+\Omega_{r, 0}\left(\frac{1}{a^{2}}-1\right)\right]^{-\frac{1}{2}}
$$

where $\Omega_{\Lambda, 0}, \Omega_{m, 0}$ and $\Omega_{r, 0}$ are the density parameters of the dark energy, matter and radiation at present time respectively. $H_{0}$ is the Hubble parameter at the present time, so Equation (18) can be written as: 


$$
\frac{\mathrm{d} t}{\mathrm{~d} a}=\frac{1}{H_{0}}\left[1-\Omega_{\Lambda, 0}\left(1-a^{2}\right)+\Omega_{m, 0}\left(\frac{1}{a}-1\right)+\Omega_{r, 0}\left(\frac{1}{a^{2}}-1\right)\right]^{-\frac{1}{2}}
$$

Substituting by Equation (19) in Equation (17), we find:

$$
d_{p}\left(t_{0}\right)=\frac{c}{H_{0}} \int_{(1+z)}^{1} \frac{1}{a}\left[1-\Omega_{\Lambda, 0}\left(1-a^{2}\right)+\Omega_{m, 0}\left(\frac{1}{a}-1\right)+\Omega_{r, 0}\left(\frac{1}{a^{2}}-1\right)\right]^{-\frac{1}{2}} \mathrm{~d} a
$$

where $1+z=\frac{1}{a\left(t_{e}\right)}, z$ is the redshift of object.

The luminosity distance $d_{L}\left(t_{0}\right)$, angular diameter distance $d_{A}\left(t_{0}\right)$ and distance modulus of the object at the present time are expressed respectively as:

$$
\begin{gathered}
d_{L}\left(t_{0}\right)=d_{p}\left(t_{0}\right)(1+z) \\
d_{A}\left(t_{0}\right)=\frac{d_{p}\left(t_{0}\right)}{1+z} \\
m-M=5 \log d_{L}+25
\end{gathered}
$$

where $m, M$ are the apparent and absolute magnitudes of the object, the distance $d_{p}, d_{L}$ and $d_{A}$ are measured in $M_{p c}$.

It is obvious from Equation (20) that the horizon distance of the universe at the present time is given by

$$
d_{h}\left(t_{0}\right)=\frac{c}{H_{0}} \int_{0}^{1} \frac{1}{a}\left[1-\Omega_{\Lambda, 0}\left(1-a^{2}\right)+\Omega_{m, 0}\left(\frac{1}{a}-1\right)+\Omega_{r, 0}\left(\frac{1}{a^{2}}-1\right)\right]^{-1 / 2} \mathrm{~d} a
$$

where $z=\infty$. The Robertson-Walker metric of space-time which describes the expansion or contraction of homogeneous and isotropic universe is given by:

$$
\mathrm{d} s^{2}=c^{2} \mathrm{~d} t^{2}-R^{2}(t)\left[\frac{\mathrm{d} r^{2}}{1-k r^{2}}+r^{2} \mathrm{~d} \theta^{2}+r^{2} \sin ^{2} \theta \mathrm{d} \varnothing^{2}\right]
$$

where $r, \theta, \varnothing$ are the comoving coordinates of a point in space. The volume of three dimensional sphere can be written as:

$$
V(t)=2 \int_{0}^{2 \pi} \mathrm{d} \varnothing \int_{0}^{\pi} \mathrm{d} \theta \int_{0}^{r_{0}} \sqrt{\operatorname{det} g_{R W}} \mathrm{~d} r
$$

where $\operatorname{det} g_{R W}$ is the determinant of the spatial part of the Robertson-Walker metric matrix and given by

$$
\operatorname{det} g_{R W}=R^{6}(t) \frac{r^{4} \sin ^{2} \theta}{\sqrt{1-k r^{2}}}
$$

Since the space of the universe is flat at the present time, hence $k=0$ and

$$
\sqrt{\operatorname{det} g_{R W}}=R^{3}\left(t_{0}\right) r^{2} \sin \theta
$$

Substituting by (28) in (26) we have

$$
\begin{gathered}
V\left(t_{0}\right)=2 R^{3}\left(t_{0}\right) \int_{0}^{2 \pi} \mathrm{d} \varnothing \int_{0}^{\pi} \sin \theta \mathrm{d} \theta \int_{0}^{r_{0}} r^{2} \mathrm{~d} r \\
V\left(t_{0}\right)=\frac{8 \pi}{3} R^{3}\left(t_{0}\right) r_{0}^{3}
\end{gathered}
$$


For flat space Equation (14) and (15)

$$
d_{p}\left(t_{0}\right)=R\left(t_{0}\right) r_{0}
$$

Substituting by Equation (30) in (29), we get at the present time:

$$
V\left(t_{0}\right)=\frac{8 \pi}{3} d_{p}^{3}\left(t_{0}\right)
$$

Similarly the volume of sphere of radius $d_{h}\left(t_{0}\right)$ is expressed as:

$$
V_{h}\left(t_{0}\right)=\frac{8 \pi}{3} d_{h}^{3}\left(t_{0}\right)
$$

Horizon distance of the universe at any given time:

$$
d_{h}(t)=\frac{c}{H_{0}} \int_{0}^{a} \frac{1}{a}\left[1-\Omega_{\Lambda, 0}\left(1-a^{2}\right)+\Omega_{m, 0}\left(\frac{1}{a}-1\right)+\Omega_{r, 0}\left(\frac{1}{a^{2}}-1\right)\right]^{-1 / 2} \mathrm{~d} a
$$

And at any time the total density of universe is given by:

$$
\rho(t)=\rho_{c, t} \Omega(t)
$$

where

$$
\begin{gathered}
\Omega(t)=\rho_{c, t}\left(\Omega_{m, t}+\Omega_{r, t}+\Omega_{\Lambda, t}\right) \\
\rho_{c, t}=\frac{3 H^{2}}{8 \pi G} \\
\Omega_{m, t}=\frac{\rho_{m, t}}{\rho_{c, t}}=\left(\frac{H_{0}}{H}\right)^{2} \frac{\Omega_{m, 0}}{a^{3}} \\
\Omega_{\Lambda, t}=\frac{\rho_{\Lambda, t}}{\rho_{c, t}}=\left(\frac{H_{0}}{H}\right)^{2} \Omega_{\Lambda, 0} \\
H(t)=\frac{H_{0}}{a}\left[1-\Omega_{\Lambda, 0}\left(1-a^{2}\right)+\Omega_{m, 0}\left(\frac{1}{a}-1\right)+\Omega_{r, 0}\left(\frac{1}{a^{2}}-1\right)\right]^{1 / 2}
\end{gathered}
$$

Hence, the total mass within the horizon-volume of the universe at any given time is expressed as:

$$
M_{h}(t)=V_{h}(t) \rho(t)
$$

Cosmic time:

$$
t=\frac{1}{H_{0}} \int_{0}^{a}\left[1-\Omega_{\Lambda, 0}\left(1-a^{2}\right)+\Omega_{m, 0}\left(\frac{1}{a}-1\right)+\Omega_{r, 0}\left(\frac{1}{a^{2}}-1\right)\right]^{1 / 2} \mathrm{~d} a
$$

This all relation of density, and mass distribution in space. Time distribution [8] define the mass distribution is a aspect of space-time expansion

There is a relation between mass and space.

According to theory of relativity.

$$
M=\frac{C^{2}}{G_{0}} \varepsilon_{o} \frac{V}{S} \rho_{s}=f(v)
$$

where $\varepsilon_{o}$ is elasticity of space-time and $f(v)=$ matter volume, $\rho_{s}$ is the density of space-time [9]. 
This equation has a clear indication about the mass and space-time co-relation and co-exit in the evolution of universe.

\section{Active Universe: By Mass-Distortion}

There is another concept about empty volume which is an empty $3 \mathrm{D}$ vacumn or gap between atoms. This is an inactive space-time. There is no any curvature or activity in space-time. [10] Empty volume is a transparent regarding space-time. This special kind of volume does not have mass and does not curve space (Figure 1).

There is number of dark matter a profile which was articulated for example [11] [12] [13] [14] [15]. These are defining that dark matter is part of expansion. So space can expand with the dark matter as galaxies were expanding with the help of energy of dark matter. So dark matter is ingredient of space, that's why matter is part of space-time. As dark matter is source of space so mass is fundamental part of space-time continuum.

\section{Energy and Temperature}

There is a relation between radiation of cosmic background and temperature. Energy order of $E \sim 10^{20} \mathrm{GeV}$ will emit during the particle's time crossing a galaxy $t_{o}=\frac{r_{o}}{v} \sim 10^{13} \mathrm{sec}$.

Hence the corresponding energy density in a halo will be $\varepsilon \approx \frac{E}{R_{O}^{3}} \sim 10^{-47} \mathrm{GeV} / \mathrm{cm}^{3} \approx 10^{-44} \mathrm{Erg} / \mathrm{cm}^{3}$, and on the basis of Stephen Boltzmann law [16]

$$
\varepsilon=\sigma T^{4}
$$

where $\sigma \sim 10^{-15} \mathrm{Erg} / \mathrm{cm}^{3} \cdot \mathrm{K}^{4}$.

This is the evidence of radiation matter relation between temperatures in space. So temperature have role of state transformation or mass-state.

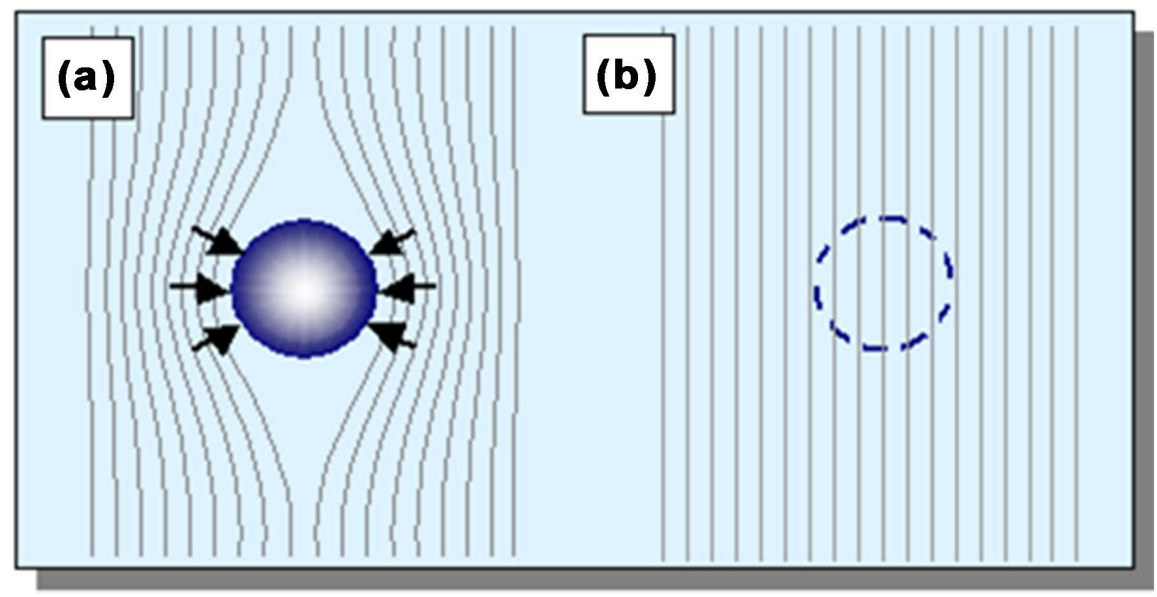

Figure 1. (a) is matter-volume that curve the space-time, and (b) does not because it is empty volume such as orbital of atom (excluding the nucleus and electrons). 
The high energy particles are traversing the universe through the cosmic microwave background [17]. This article defines the mass distribution in universe that is the part of space. So matter of radiation is spread out in whole universe.

In revolutionary paper of Albert Einstein explain the famous relation of mass and energy that is a significant part of space-time relation with mass-energy as any matter can be form energy. So mass will be energy [18].

So space-time-mass continuum is reliable aspect of universe that helps to explain the space-continuum.

\section{Conclusion}

In this paper, I am trying to present that mass is also an undividable part of space-time because without mass there is no any type of action or phenomenon occur in space-time. As space only expands by the help of matter either it is positive or negative. So space is a consequence of matter or energy. So space-time mass is a continuum of an active universe. And distortion in space occurs due to the mass in space. There is a fixed mass relation between space-time, in other words, mass is a property of space-time continuum for a static universe, but for an active universe (Section 4) mass should be a dimension of universe.

\section{References}

[1] Palacios, A.F. (2017) About the Mass. Open Access Library Journal, 4, e2835.

[2] Einstein, A. (translated by Satyendra Nath Bose) (1915) The Principle of Relativity. Original Papers by A. Einstein and H. Minkowski, University of Calcutta, 89-163.

[3] Bernard, S. (2003) Gravity from the Ground up: An Entry Guide to Gravity and General Relativity. Cambridge University Press, Cambridge, 462 p.

[4] Nikouravan, B. and Rawal, J.J. (2013) Mass as the Fifth Dimension of the Universe. International Journal of Astronomy and Astrophysics, 3, 256-259.

[5] Schwarzchild, K. (1916) On the Gravitational Field of Mass Point, According to Einstein's Theory. Sitzungsber. Preuss. Akad. Wiss., Phys. Math. Kl, 3, 189-196.

[6] Baurov, Y.A. (1998) Structure of Physical Space and New Method of Obtaining Energy (Theory, Experiment, Applications). Moscow, Krechet, in Russian.

[7] Meessen, A. (2017) Accelerating Expansion of Space, Dark Matter, Dark Energy and Big Bang Process. Journal of Modern Physics, 8, 251-267. https://doi.org/10.4236/jmp.2017.82017

[8] Bukhari, F.A. (2015) Distribution of Mass and Energy in Five General Cosmic Models. International Journal of Astronomy and Astrophysics, 5, 20-27. https://doi.org/10.4236/ijaa.2015.51004

[9] Jeromi, J. and Jerome, F. (2005) New Viewpoint of General Relativity That Explains Mass and Gravity and Some Enigmas of Modan Physics. Science and Tech. Neotrinos NIPI 238268.

[10] Einstein, A. and Grassmann, M. (1913) Outline of a Generalised Theory of Relativity and of a Theory of Gravitation. Zeitschrift Fur Mathematik and Physik, 62, 225-261.

[11] Klypin, A.A., Kravstaw, A.V., Bulcok, J., et al. (2001) Resolving the Structure of Cold Dark Matter Halos II. The Astrophysical Journal, 554, 903. 
https://doi.org/10.1086/321400

[12] Einasto, J. (2000) Dark Matter and Large Scale Structure. astro-ph1001216vl.

[13] Treuand, T. and Koopmans, V.E. (2004) Massive Dark Matter Halos \& Early-Type Galaxies to $\mathrm{z}=1$. The Astro. $Z ., 611,339$.

[14] Weber, M. and de Boer, W. (2010) Determination of the Local Dark Matter Density in Our Galaxy. Astronomy \& Astrophysics, 509, Article Number: A25. https://doi.org/10.1051/0004-6361/200913381

[15] Catena, R. and Ullio, P. (2009) A Novel Determination of the Local Dark Matter Density. Journal of Cosmology and Astroparticle Physics, 8, Astro.ph C0/0907.0018v2.

[16] Gurevtch, L.E. and Chernin (1978) Vvedeniye-v kos mogoniyu. Nauka, Moscow.

[17] Chechin, L.M. (2013) The Cherenkov Radiation in the Galaxy's Halo of Dark Matter. International Journal of Astronomy and Astrophysics, 3, 285-290. https://doi.org/10.4236/ijaa.2013.33034

[18] Einstein, A. (1905) Zur Elektrodynamik bewegter Körper. Annalen der Physik, 322, 891. https://doi.org/10.1002/andp.19053221004 